\title{
On the edge-Wiener index of the disjunctive product of simple graphs
}

\author{
M. Azari* and A. Iranmanesh
}

Communicated by D. Simson

\begin{abstract}
A BStRACT. The edge-Wiener index of a simple connected graph $G$ is defined as the sum of distances between all pairs of edges of $G$ where the distance between two edges in $G$ is the distance between the corresponding vertices in the line graph of $G$. In this paper, we study the edge-Wiener index under the disjunctive product of graphs and apply our results to compute the edge-Wiener index for the disjunctive product of paths and cycles.
\end{abstract}

\section{Introduction}

Throughout this paper, we consider connected finite graphs without any loops or multiple edges. Let $G$ be such a graph with vertex set $V(G)$ and edge set $E(G)$. A topological index (also known as graph invariant) is any function on a graph that does not depend on a labeling of its vertices. Several hundreds of different invariants have been employed to date with various degrees of success in QSAR/QSPR studies. We refer the reader to [12] for review.

The oldest topological index is the one put forward in 1947 by Harold Wiener [23] nowadays referred to as the Wiener index. Wiener used his index for the calculation of the boiling points of alkanes. The Wiener

${ }^{*}$ Corresponding author.

2010 MSC: 05C76, 05C12, 05C38.

Key words and phrases: distance in graphs, edge-Wiener index, disjunctive product of graphs. 
index $W(G)$ of a graph $G$ is defined as the sum of distances between all pairs of vertices of $G$,

$$
W(G)=\sum_{\{u, v\} \subseteq V(G)} d(u, v \mid G),
$$

where $d(u, v \mid G)$ denotes the distance between the vertices $u$ and $v$ of $G$ which is defined as the length of any shortest path in $G$ connecting them. We denote $d(u, v \mid G)$ simply by $d(u, v)$ when no ambiguity is present. Details on the mathematical properties of the Wiener index and its applications in chemistry can be found in $[8,10,11,13-15]$.

Motivated by definition of the Wiener index, the edge-Wiener index was introduced based on distance between all pairs of edges in a graph in $2009[9,19,21]$. The edge-Wiener index of a graph $G$ is defined as

$$
W_{e}(G)=\sum_{\{f, g\} \subseteq E(G)} d_{e}(f, g \mid G)
$$

where $d_{e}(f, g \mid G)$ denotes the distance between the edges $f$ and $g$ of $G$ which is defined as the ordinary distance between the corresponding vertices in the line graph $L(G)$ of $G$. So, $W_{e}(G)=W(L(G))$. It has been proved that [19], for each pair of edges $f=u v$ and $g=z t$ of $G$,

$$
d_{e}(f, g \mid G)= \begin{cases}\min \{d(u, z), d(u, t), d(v, z), d(v, t)\}+1 & \text { if } f \neq g, \\ 0 & \text { if } f=g .\end{cases}
$$

For details on the theory of the edge-Wiener index and its applications see $[2,22,24]$ and specially the recent paper [18].

Many graphs are composed of simpler graphs via various graph operations also known as graph products. These composite graphs have more complicated structures than their components. So, in general, computing their topological invariants is more difficult than computing the topological invariants of their components. So, it is important to understand how certain invariants of such composite graphs are related to the corresponding invariants of their components. The edge-Wiener index of some graph operations have been computed before [1,3-7]. In this paper, we study the behavior of the edge-Wiener index under the disjunctive product of graphs and apply our results to compute the edge-Wiener index for the disjunctive product of paths and cycles. We refer the reader to [17] for details on the properties and applications of graph operations. 


\section{Definitions and preliminaries}

For a simple connected graph $G$, let $N_{G}(u)$ denote the open neighborhood of a vertex $u$ in $G$ which is the set of all vertices of $G$ adjacent with $u$. The cardinality of $N_{G}(u)$ is called the degree of $u$ in $G$ and denoted by $d_{G}(u)$. If there is no confusion, we simply use $N(u)$ and $d(u)$ instead of $N_{G}(u)$ and $d_{G}(u)$, respectively. Let $\Delta(G)$ denote the number of all triangles (3-cycles) in $G$ and $M_{1}(G)$ denote the first Zagreb index of $G$ which is one the oldest topological indices introduced by Gutman and Trinajstić [16] as follow.

$$
M_{1}(G)=\sum_{u \in V(G)} d(u)^{2}=\sum_{u v \in E(G)}(d(u)+d(v)) .
$$

It is easy to check that,

$$
\sum_{u v \in E(G)}|N(u) \cap N(v)|=3 \Delta(G)
$$

and

$$
\sum_{u, v \in V(G)}|N(u) \cap N(v)|=M_{1}(G) .
$$

Using the inclusion-exclusion principle and then (1), (2), and (3), one can easily get the following equations.

$$
\sum_{u v \in E(G)}|N(u) \cup N(v)|=M_{1}(G)-3 \Delta(G)
$$

and

$$
\sum_{u, v \in V(G)}|N(u) \cup N(v)|=4 n e-M_{1}(G),
$$

where $n$ and $e$ denote the order and size of the graph $G$, respectively.

Here, we introduce some useful notations which will be used throughout the paper.

$$
\begin{aligned}
\nu(G) & =\sum_{u v \in E(G)}|N(u) \cup N(v)|^{2}, \\
\nu^{*}(G) & =\sum_{u, v \in V(G)}|N(u) \cup N(v)|^{2},
\end{aligned}
$$




$$
\mu(G)=\sum_{u v \in E(G)} \sum_{z \in V(G) \backslash(N(u) \cup N(v))}|N(z) \backslash(N(u) \cup N(v))|,
$$

and

$$
\mu^{*}(G)=\sum_{u, v \in V(G)} \sum_{z \in V(G) \backslash(N(u) \cup N(v))}|N(z) \backslash(N(u) \cup N(v))| .
$$

\section{Results and discussion}

Let $G_{1}$ and $G_{2}$ be two simple connected graphs. We denote by $V\left(G_{i}\right)$ and $E\left(G_{i}\right)$, the vertex set and edge set of $G_{i}$, respectively, where $i \in\{1,2\}$. The disjunctive product $G_{1} \vee G_{2}$ of graphs $G_{1}$ and $G_{2}$ is a graph with the vertex set $V\left(G_{1}\right) \times V\left(G_{2}\right)$ and two vertices $\left(u_{1}, u_{2}\right)$ and $\left(v_{1}, v_{2}\right)$ of $G_{1} \vee G_{2}$ are adjacent if and only if $u_{1}$ and $v_{1}$ are adjacent in $G_{1}$ or $u_{2}$ and $v_{2}$ are adjacent in $G_{2}$. The disjunctive product of two graphs is also known as their co-normal product or OR product. The distance between the vertices $u=\left(u_{1}, u_{2}\right)$ and $v=\left(v_{1}, v_{2}\right)$ of $G_{1} \vee G_{2}$ is given by

$$
d\left(u, v \mid G_{1} \vee G_{2}\right)= \begin{cases}0 & \text { if } u_{1}=v_{1}, u_{2}=v_{2}, \\ 1 & \text { if } u_{1} v_{1} \in E\left(G_{1}\right) \text { or } u_{2} v_{2} \in E\left(G_{2}\right), \\ 2 & \text { otherwise. }\end{cases}
$$

In this section, we compute the edge-Wiener index of the disjunctive product of $G_{1}$ and $G_{2}$. Throughout the section, for notational convenience, we let $G=G_{1} \vee G_{2}$ be the disjunctive product of a pair of graphs $G_{1}$ and $G_{2}$, and $n_{1}, e_{1}, n_{2}, e_{2}$ denote the order of $G_{1}$, size of $G_{1}$, order of $G_{2}$, size of $G_{2}$, respectively.

At first, we consider three subsets of $E(G)$ as follows.

$$
\begin{aligned}
& E_{1}=\left\{\left(u_{1}, u_{2}\right)\left(v_{1}, v_{2}\right) \mid u_{1} v_{1} \in E\left(G_{1}\right), u_{2}, v_{2} \in V\left(G_{2}\right)\right\}, \\
& E_{2}=\left\{\left(u_{1}, u_{2}\right)\left(v_{1}, v_{2}\right) \mid u_{2} v_{2} \in E\left(G_{2}\right), u_{1}, v_{1} \in V\left(G_{1}\right)\right\}, \\
& E_{3}=\left\{\left(u_{1}, u_{2}\right)\left(v_{1}, v_{2}\right) \mid u_{1} v_{1} \in E\left(G_{1}\right), u_{2} v_{2} \in E\left(G_{2}\right)\right\} .
\end{aligned}
$$

It is clear that, $E(G)=\bigcup_{i=1}^{3} E_{i}$ and

$$
|E(G)|=\left|E_{1}\right|+\left|E_{2}\right|-2\left|E_{3}\right|=e_{1} n_{2}^{2}+e_{2} n_{1}^{2}-2 e_{1} e_{2} .
$$

Since all distinct vertices of $G$ are either at distance 1 or 2 , so all distinct edges of $G$ are either at distance 1,2, or 3. Therefore, we can partition the set of all pairs of edges of $G$ into three sets as follows.

$$
A=\left\{\{f, g\} \mid d_{e}(f, g \mid G)=1\right\},
$$




$$
\begin{aligned}
& B=\left\{\{f, g\} \mid d_{e}(f, g \mid G)=2\right\}, \\
& C=\left\{\{f, g\} \mid d_{e}(f, g \mid G)=3\right\} .
\end{aligned}
$$

In order to find the edge-Wiener index of $G$, we should compute the cardinality of the above sets. It is clear that,

$$
|A|+|B|+|C|=\left(\begin{array}{c}
|E(G)| \\
2
\end{array}\right)=\left(\begin{array}{c}
e_{1} n_{2}^{2}+e_{2} n_{1}^{2}-2 e_{1} e_{2} \\
2
\end{array}\right) .
$$

By (11), it is enough to find the cardinality of the sets $A$ and $C$.

In the following proposition, we compute the cardinality of the set $A$.

Proposition 1. The cardinality of the set $A$ is given by

$$
\begin{aligned}
|A|=\frac{1}{2}[ & n_{2}\left(n_{2}^{2}-4 e_{2}\right) M_{1}\left(G_{1}\right)+n_{1}\left(n_{1}^{2}-4 e_{1}\right) M_{1}\left(G_{2}\right) \\
& \left.\quad+M_{1}\left(G_{1}\right) M_{1}\left(G_{2}\right)+8 n_{1} n_{2} e_{1} e_{2}-2\left(e_{1} n_{2}^{2}+e_{2} n_{1}^{2}-2 e_{1} e_{2}\right)\right] .
\end{aligned}
$$

Proof. Clearly, $A$ is the set of all pairs of adjacent edges of $G$. So

$$
|A|=\sum_{u \in V(G)}\left(\begin{array}{c}
d(u) \\
2
\end{array}\right)=\frac{1}{2} \sum_{u \in V(G)}\left(d(u)^{2}-d(u)\right)=\frac{1}{2} M_{1}(G)-|E(G)| .
$$

By Theorem 3 in [20], the first Zagreb index of the disjunctive product of $G_{1}$ and $G_{2}$ is given by

$$
\begin{aligned}
M_{1}(G)= & n_{2}\left(n_{2}^{2}-4 e_{2}\right) M_{1}\left(G_{1}\right)+n_{1}\left(n_{1}^{2}-4 e_{1}\right) M_{1}\left(G_{2}\right) \\
& +M_{1}\left(G_{1}\right) M_{1}\left(G_{2}\right)+8 n_{1} n_{2} e_{1} e_{2} .
\end{aligned}
$$

Now using (10), we can get (12).

Now we start to find the cardinality of the set $C$. Suppose $f=$ $\left(u_{1}, u_{2}\right)\left(v_{1}, v_{2}\right)$ is an arbitrary edge of $G$ and let $C(f)$ be the set of all edges of $G$ which are at distance 3 from $f$,

$$
C(f)=\left\{g \in E(G) \mid d_{e}(f, g \mid G)=3\right\} .
$$

In the following lemma, we compute the cardinality of the set $C(f)$.

Lemma 1. For every arbitrary edge $f=\left(u_{1}, u_{2}\right)\left(v_{1}, v_{2}\right)$ of $G$, the cardinality of the set $C(f)$ is given by

$$
|C(f)|=\frac{1}{2}\left[\left(n_{2}-\left|N\left(u_{2}\right) \cup N\left(v_{2}\right)\right|\right)^{2}\right.
$$




$$
\begin{gathered}
\times \sum_{z_{1} \in V\left(G_{1}\right) \backslash\left(N\left(u_{1}\right) \cup N\left(v_{1}\right)\right)}\left|N\left(z_{1}\right) \backslash\left(N\left(u_{1}\right) \cup N\left(v_{1}\right)\right)\right| \\
+\left(n_{1}-\left|N\left(u_{1}\right) \cup N\left(v_{1}\right)\right|\right)^{2} \\
\times \sum_{z_{2} \in V\left(G_{2}\right) \backslash\left(N\left(u_{2}\right) \cup N\left(v_{2}\right)\right)}\left|N\left(z_{2}\right) \backslash\left(N\left(u_{2}\right) \cup N\left(v_{2}\right)\right)\right| \\
-\sum_{z_{1} \in V\left(G_{1}\right) \backslash\left(N\left(u_{1}\right) \cup N\left(v_{1}\right)\right)}\left|N\left(z_{1}\right) \backslash\left(N\left(u_{1}\right) \cup N\left(v_{1}\right)\right)\right| \\
\left.\times \sum_{z_{2} \in V\left(G_{2}\right) \backslash\left(N\left(u_{2}\right) \cup N\left(v_{2}\right)\right)}\left|N\left(z_{2}\right) \backslash\left(N\left(u_{2}\right) \cup N\left(v_{2}\right)\right)\right|\right] .
\end{gathered}
$$

Proof. Let $f=\left(u_{1}, u_{2}\right)\left(v_{1}, v_{2}\right)$ be an arbitrary edge of $G$ and let $g=$ $\left(z_{1}, z_{2}\right)\left(t_{1}, t_{2}\right)$ be an arbitrary element of $C(f)$. By definition of the distance $d_{e}$, we have

$$
\begin{gathered}
1+\min \left\{d\left(\left(u_{1}, u_{2}\right),\left(z_{1}, z_{2}\right)\right), d\left(\left(u_{1}, u_{2}\right),\left(t_{1}, t_{2}\right)\right), d\left(\left(v_{1}, v_{2}\right),\left(z_{1}, z_{2}\right)\right),\right. \\
\left.d\left(\left(v_{1}, v_{2}\right),\left(t_{1}, t_{2}\right)\right)\right\}=d_{e}(f, g \mid G)=3 .
\end{gathered}
$$

Hence,

$$
\begin{aligned}
\min & \left\{d\left(\left(u_{1}, u_{2}\right),\left(z_{1}, z_{2}\right)\right), d\left(\left(u_{1}, u_{2}\right),\left(t_{1}, t_{2}\right)\right), d\left(\left(v_{1}, v_{2}\right),\left(z_{1}, z_{2}\right)\right),\right. \\
& \left.d\left(\left(v_{1}, v_{2}\right),\left(t_{1}, t_{2}\right)\right)\right\}=2 .
\end{aligned}
$$

Since all distinct vertices of $G$ are either at distance 1 or 2 , so

$$
\begin{gathered}
d\left(\left(u_{1}, u_{2}\right),\left(z_{1}, z_{2}\right)\right)=d\left(\left(u_{1}, u_{2}\right),\left(t_{1}, t_{2}\right)\right)=d\left(\left(v_{1}, v_{2}\right),\left(z_{1}, z_{2}\right)\right) \\
=d\left(\left(v_{1}, v_{2}\right),\left(t_{1}, t_{2}\right)\right)=2 .
\end{gathered}
$$

This implies that, $z_{i}$ and $t_{i}$ are adjacent neither to $u_{i}$ nor to $v_{i}$ in $G_{i}$, where $i \in\{1,2\}$. Consequently,

$$
\begin{aligned}
&|C(f)|=\frac{1}{2} \sum_{z_{1} \in V\left(G_{1}\right) \backslash\left(N\left(u_{1}\right) \cup N\left(v_{1}\right)\right)} \sum_{z_{2} \in V\left(G_{2}\right) \backslash\left(N\left(u_{2}\right) \cup N\left(v_{2}\right)\right)}\left[\mid N\left(z_{1}\right) \backslash\left(N\left(u_{1}\right)\right.\right. \\
&\left.\cup N\left(v_{1}\right)\right) \mid\left(n_{2}-\left|N\left(u_{2}\right) \cup N\left(v_{2}\right)\right|\right) \\
&+\left|N\left(z_{2}\right) \backslash\left(N\left(u_{2}\right) \cup N\left(v_{2}\right)\right)\right|\left(n_{1}-\left|N\left(u_{1}\right) \cup N\left(v_{1}\right)\right|\right) \\
&\left.-\left|N\left(z_{1}\right) \backslash\left(N\left(u_{1}\right) \cup N\left(v_{1}\right)\right)\right|\left|N\left(z_{2}\right) \backslash\left(N\left(u_{2}\right) \cup N\left(v_{2}\right)\right)\right|\right] . \\
&=\frac{1}{2}\left[\sum_{z_{1} \in V\left(G_{1}\right) \backslash\left(N\left(u_{1}\right) \cup N\left(v_{1}\right)\right)}\left|N\left(z_{1}\right) \backslash\left(N\left(u_{1}\right) \cup N\left(v_{1}\right)\right)\right|\right.
\end{aligned}
$$




$$
\begin{gathered}
\sum_{z_{2} \in V\left(G_{2}\right) \backslash\left(N\left(u_{2}\right) \cup N\left(v_{2}\right)\right)}\left(n_{2}-\left|N\left(u_{2}\right) \cup N\left(v_{2}\right)\right|\right) \\
+\sum_{z_{2} \in V\left(G_{2}\right) \backslash\left(N\left(u_{2}\right) \cup N\left(v_{2}\right)\right)}\left|N\left(z_{2}\right) \backslash\left(N\left(u_{2}\right) \cup N\left(v_{2}\right)\right)\right| \\
\sum_{z_{1} \in V\left(G_{1}\right) \backslash\left(N\left(u_{1}\right) \cup N\left(v_{1}\right)\right)}\left(n_{1}-\left|N\left(u_{1}\right) \cup N\left(v_{1}\right)\right|\right) \\
\sum_{z_{1} \in V\left(G_{1}\right) \backslash\left(N\left(u_{1}\right) \cup N\left(v_{1}\right)\right)}\left|N\left(z_{1}\right) \backslash\left(N\left(u_{1}\right) \cup N\left(v_{1}\right)\right)\right| \\
\left.\sum_{z_{2} \in V\left(G_{2}\right) \backslash\left(N\left(u_{2}\right) \cup N\left(v_{2}\right)\right)}\left|N\left(z_{2}\right) \backslash\left(N\left(u_{2}\right) \cup N\left(v_{2}\right)\right)\right|\right] .
\end{gathered}
$$

Now, (13) is obtained after simplifying the above expression.

Let $f=\left(u_{1}, u_{2}\right)\left(v_{1}, v_{2}\right)$ be an edge of $G$. Then, $\left(u_{1}, v_{2}\right)\left(v_{1}, u_{2}\right)$ is also an edge of $G$. We denote the edge $\left(u_{1}, v_{2}\right)\left(v_{1}, u_{2}\right)$ by $\bar{f}$.

Lemma 2. For every arbitrary edge $f=\left(u_{1}, u_{2}\right)\left(v_{1}, v_{2}\right)$ of $G,|C(\bar{f})|=$ $|C(f)|$.

Proof. The cardinality of $C(\bar{f})$ can easily be obtained by changing the role of the vertices $u_{2}$ and $v_{2}$ in (13). On the other hand, one can easily check that changing the role of these two vertices does not influence the result. So $|C(\bar{f})|=|C(f)|$.

In the following proposition, we obtain the cardinality of the set $C$.

Proposition 2. The cardinality of the set $C$ is given by

$$
\begin{aligned}
|C|=\frac{1}{4}[( & n_{2}^{2}\left(n_{2}^{2}-10 e_{2}\right)+\nu^{*}\left(G_{2}\right)-2 \nu\left(G_{2}\right) \\
& \left.+6 n_{2}\left(M_{1}\left(G_{2}\right)-2 \Delta\left(G_{2}\right)\right)\right) \mu\left(G_{1}\right) \\
& +\left(n_{1}^{2}\left(n_{1}^{2}-10 e_{1}\right)+\nu^{*}\left(G_{1}\right)-2 \nu\left(G_{1}\right)\right. \\
& \left.+6 n_{1}\left(M_{1}\left(G_{1}\right)-2 \Delta\left(G_{1}\right)\right)\right) \mu\left(G_{2}\right) \\
& +\left(n_{2}^{2} e_{2}+\nu\left(G_{2}\right)-2 n_{2}\left(M_{1}\left(G_{2}\right)-3 \Delta\left(G_{2}\right)\right)\right) \mu^{*}\left(G_{1}\right) \\
& +\left(n_{1}^{2} e_{1}+\nu\left(G_{1}\right)-2 n_{1}\left(M_{1}\left(G_{1}\right)-3 \Delta\left(G_{1}\right)\right)\right) \mu^{*}\left(G_{2}\right) \\
& \left.-\mu\left(G_{1}\right) \mu^{*}\left(G_{2}\right)-\mu\left(G_{2}\right) \mu^{*}\left(G_{1}\right)+2 \mu\left(G_{1}\right) \mu\left(G_{2}\right)\right] .
\end{aligned}
$$


Proof. For every $f \in E(G)$, there exist $|C(f)|$ elements in $C$. Furthermore, for every pair of edges $f, g$ in $G, g \in C(f)$ if and only if $f \in C(g)$. Hence,

$$
\begin{aligned}
|C|= & \frac{1}{2} \sum_{f \in E(G)}|C(f)|=\frac{1}{2}\left[\sum_{f \in E_{1}}|C(f)|+\sum_{f \in E_{2}}|C(f)|\right. \\
& \left.-\sum_{f \in E_{3}}(|C(f)|+|C(\bar{f})|)\right] .
\end{aligned}
$$

Using Lemma 2, we obtain

$$
|C|=\frac{1}{2}\left[\sum_{f \in E_{1}}|C(f)|+\sum_{f \in E_{2}}|C(f)|-2 \sum_{f \in E_{3}}|C(f)|\right] .
$$

Now, we compute $\sum_{f \in E_{i}}|C(f)|$, for every $i \in\{1,2,3\}$.

By definition of the set $E_{1}$ and (13), we have

$$
\begin{aligned}
\sum_{f \in E_{1}}|C(f)|= & \frac{1}{2} \sum_{u_{1} v_{1} \in E\left(G_{1}\right)} \sum_{u_{2}, v_{2} \in V\left(G_{2}\right)}\left[\left(n_{2}-\left|N\left(u_{2}\right) \cup N\left(v_{2}\right)\right|\right)^{2}\right. \\
& \sum_{z_{1} \in V\left(G_{1}\right) \backslash\left(N\left(u_{1}\right) \cup N\left(v_{1}\right)\right)}\left|N\left(z_{1}\right) \backslash\left(N\left(u_{1}\right) \cup N\left(v_{1}\right)\right)\right| \\
& +\left(n_{1}-\left|N\left(u_{1}\right) \cup N\left(v_{1}\right)\right|\right)^{2} \\
& \sum_{z_{2} \in V\left(G_{2}\right) \backslash\left(N\left(u_{2}\right) \cup N\left(v_{2}\right)\right)}\left|N\left(z_{2}\right) \backslash\left(N\left(u_{2}\right) \cup N\left(v_{2}\right)\right)\right| \\
& -\sum_{z_{1} \in V\left(G_{1}\right) \backslash\left(N\left(u_{1}\right) \cup N\left(v_{1}\right)\right)}\left|N\left(z_{1}\right) \backslash\left(N\left(u_{1}\right) \cup N\left(v_{1}\right)\right)\right| \\
& \left.\sum_{z_{2} \in V\left(G_{2}\right) \backslash\left(N\left(u_{2}\right) \cup N\left(v_{2}\right)\right)}\left|N\left(z_{2}\right) \backslash\left(N\left(u_{2}\right) \cup N\left(v_{2}\right)\right)\right|\right] .
\end{aligned}
$$

By simplifying the above expression, we obtain

$$
\begin{aligned}
& \sum_{f \in E_{1}}|C(f)|=\frac{1}{2}\left[\sum_{u_{2}, v_{2} \in V\left(G_{2}\right)}\left(n_{2}-\left|N\left(u_{2}\right) \cup N\left(v_{2}\right)\right|\right)^{2}\right. \\
& \quad \sum_{u_{1} v_{1} \in E\left(G_{1}\right)} \sum_{z_{1} \in V\left(G_{1}\right) \backslash\left(N\left(u_{1}\right) \cup N\left(v_{1}\right)\right)}\left|N\left(z_{1}\right) \backslash\left(N\left(u_{1}\right) \cup N\left(v_{1}\right)\right)\right| \\
& +\sum_{u_{1} v_{1} \in E\left(G_{1}\right)}\left(n_{1}-\left|N\left(u_{1}\right) \cup N\left(v_{1}\right)\right|\right)^{2} \\
& \quad \sum_{u_{2}, v_{2} \in V\left(G_{2}\right)} \sum_{z_{2} \in V\left(G_{2}\right) \backslash\left(N\left(u_{2}\right) \cup N\left(v_{2}\right)\right)}\left|N\left(z_{2}\right) \backslash\left(N\left(u_{2}\right) \cup N\left(v_{2}\right)\right)\right|
\end{aligned}
$$




$$
\begin{gathered}
-\sum_{u_{1} v_{1} \in E\left(G_{1}\right)} \sum_{z_{1} \in V\left(G_{1}\right) \backslash\left(N\left(u_{1}\right) \cup N\left(v_{1}\right)\right)}\left|N\left(z_{1}\right) \backslash\left(N\left(u_{1}\right) \cup N\left(v_{1}\right)\right)\right| \\
\left.\sum_{u_{2}, v_{2} \in V\left(G_{2}\right)} \sum_{z_{2} \in V\left(G_{2}\right) \backslash\left(N\left(u_{2}\right) \cup N\left(v_{2}\right)\right)}\left|N\left(z_{2}\right) \backslash\left(N\left(u_{2}\right) \cup N\left(v_{2}\right)\right)\right|\right] .
\end{gathered}
$$

Now using (4)-(9), we obtain

$$
\begin{aligned}
\sum_{f \in E_{1}}|C(f)|= & \frac{1}{2}\left[\left(n_{2}^{4}+\nu^{*}\left(G_{2}\right)-2 n_{2}\left(4 n_{2} e_{2}-M_{1}\left(G_{2}\right)\right)\right) \mu\left(G_{1}\right)\right. \\
& +\left(n_{1}^{2} e_{1}+\nu\left(G_{1}\right)-2 n_{1}\left(M_{1}\left(G_{1}\right)-3 \Delta\left(G_{1}\right)\right)\right) \mu^{*}\left(G_{2}\right) \\
& \left.-\mu\left(G_{1}\right) \mu^{*}\left(G_{2}\right)\right] .
\end{aligned}
$$

By definition of the set $E_{2}$ and (13), we have

$$
\begin{aligned}
\sum_{f \in E_{2}}|C(f)|= & \frac{1}{2} \sum_{u_{2} v_{2} \in E\left(G_{2}\right)} \sum_{u_{1}, v_{1} \in V\left(G_{1}\right)}\left[\left(n_{2}-\left|N\left(u_{2}\right) \cup N\left(v_{2}\right)\right|\right)^{2}\right. \\
& \sum_{z_{1} \in V\left(G_{1}\right) \backslash\left(N\left(u_{1}\right) \cup N\left(v_{1}\right)\right)}\left|N\left(z_{1}\right) \backslash\left(N\left(u_{1}\right) \cup N\left(v_{1}\right)\right)\right| \\
& +\left(n_{1}-\left|N\left(u_{1}\right) \cup N\left(v_{1}\right)\right|\right)^{2} \\
& \sum_{z_{2} \in V\left(G_{2}\right) \backslash\left(N\left(u_{2}\right) \cup N\left(v_{2}\right)\right)}\left|N\left(z_{2}\right) \backslash\left(N\left(u_{2}\right) \cup N\left(v_{2}\right)\right)\right| \\
& -\sum_{z_{1} \in V\left(G_{1}\right) \backslash\left(N\left(u_{1}\right) \cup N\left(v_{1}\right)\right)}\left|N\left(z_{1}\right) \backslash\left(N\left(u_{1}\right) \cup N\left(v_{1}\right)\right)\right| \\
& \left.\sum_{z_{2} \in V\left(G_{2}\right) \backslash\left(N\left(u_{2}\right) \cup N\left(v_{2}\right)\right)}\left|N\left(z_{2}\right) \backslash\left(N\left(u_{2}\right) \cup N\left(v_{2}\right)\right)\right|\right] .
\end{aligned}
$$

By symmetry, we obtain

$$
\begin{aligned}
\sum_{f \in E_{2}}|C(f)|= & \frac{1}{2}\left[\left(n_{1}^{4}+\nu^{*}\left(G_{1}\right)-2 n_{1}\left(4 n_{1} e_{1}-M_{1}\left(G_{1}\right)\right)\right) \mu\left(G_{2}\right)\right. \\
& +\left(n_{2}^{2} e_{2}+\nu\left(G_{2}\right)-2 n_{2}\left(M_{1}\left(G_{2}\right)-3 \Delta\left(G_{2}\right)\right)\right) \mu^{*}\left(G_{1}\right) \\
& \left.-\mu\left(G_{2}\right) \mu^{*}\left(G_{1}\right)\right] .
\end{aligned}
$$

By definition of the set $E_{3}$ and (13), we have

$$
\sum_{f \in E_{3}}|C(f)|=\frac{1}{2} \sum_{u_{1} v_{1} \in E\left(G_{1}\right)} \sum_{u_{2} v_{2} \in E\left(G_{2}\right)}\left[\left(n_{2}-\left|N\left(u_{2}\right) \cup N\left(v_{2}\right)\right|\right)^{2}\right.
$$




$$
\begin{aligned}
& \sum_{z_{1} \in V\left(G_{1}\right) \backslash\left(N\left(u_{1}\right) \cup N\left(v_{1}\right)\right)}\left|N\left(z_{1}\right) \backslash\left(N\left(u_{1}\right) \cup N\left(v_{1}\right)\right)\right| \\
& +\left(n_{1}-\left|N\left(u_{1}\right) \cup N\left(v_{1}\right)\right|\right)^{2} \\
& \sum_{z_{2} \in V\left(G_{2}\right) \backslash\left(N\left(u_{2}\right) \cup N\left(v_{2}\right)\right)}\left|N\left(z_{2}\right) \backslash\left(N\left(u_{2}\right) \cup N\left(v_{2}\right)\right)\right| \\
& -\sum_{z_{1} \in V\left(G_{1}\right) \backslash\left(N\left(u_{1}\right) \cup N\left(v_{1}\right)\right)}\left|N\left(z_{1}\right) \backslash\left(N\left(u_{1}\right) \cup N\left(v_{1}\right)\right)\right| \\
& \left.\sum_{z_{2} \in V\left(G_{2}\right) \backslash\left(N\left(u_{2}\right) \cup N\left(v_{2}\right)\right)}\left|N\left(z_{2}\right) \backslash\left(N\left(u_{2}\right) \cup N\left(v_{2}\right)\right)\right|\right] .
\end{aligned}
$$

Using (4), (6), and (8), we obtain

$$
\begin{aligned}
\sum_{f \in E_{3}}|C(f)|= & \frac{1}{2}\left[\left(n_{2}^{2} e_{2}+\nu\left(G_{2}\right)-2 n_{2}\left(M_{1}\left(G_{2}\right)-3 \Delta\left(G_{2}\right)\right)\right) \mu\left(G_{1}\right)\right. \\
& +\left(n_{1}^{2} e_{1}+\nu\left(G_{1}\right)-2 n_{1}\left(M_{1}\left(G_{1}\right)-3 \Delta\left(G_{1}\right)\right)\right) \mu\left(G_{2}\right) \\
& \left.-\mu\left(G_{1}\right) \mu\left(G_{2}\right)\right] .
\end{aligned}
$$

Now by (15)-(18), we can get (14).

Now, we are ready to compute the edge-Wiener index of the disjunctive product of $G_{1}$ and $G_{2}$.

Theorem 1. Assume that $G_{1}$ and $G_{2}$ are simple connected graphs, $G_{1} \vee G_{2}$ is the disjunctive product of $G_{1}$ and $G_{2}$, and $n_{1}, e_{1}, n_{2}, e_{2}$ denote the order of $G_{1}$, size of $G_{1}$, order of $G_{2}$, size of $G_{2}$, respectively. Under the notation introduced earlier, the edge-Wiener index $W_{e}\left(G_{1} \vee G_{2}\right)$ of the disjunctive product $G_{1} \vee G_{2}$ of $G_{1}$ and $G_{2}$ is given by

$$
\begin{aligned}
& W_{e}\left(G_{1} \vee G_{2}\right)=\frac{1}{4}\left[\left(n_{2}^{2}\left(n_{2}^{2}-10 e_{2}\right)+\nu^{*}\left(G_{2}\right)-2 \nu\left(G_{2}\right)\right.\right. \\
& \left.\quad+6 n_{2}\left(M_{1}\left(G_{2}\right)-2 \Delta\left(G_{2}\right)\right)\right) \mu\left(G_{1}\right) \\
& \quad+\left(n_{1}^{2}\left(n_{1}^{2}-10 e_{1}\right)+\nu^{*}\left(G_{1}\right)-2 \nu\left(G_{1}\right)\right. \\
& \left.\quad+6 n_{1}\left(M_{1}\left(G_{1}\right)-2 \Delta\left(G_{1}\right)\right)\right) \mu\left(G_{2}\right) \\
& \quad+\left(n_{2}^{2} e_{2}+\nu\left(G_{2}\right)-2 n_{2}\left(M_{1}\left(G_{2}\right)-3 \Delta\left(G_{2}\right)\right)\right) \mu^{*}\left(G_{1}\right) \\
& +\left(n_{1}^{2} e_{1}+\nu\left(G_{1}\right)-2 n_{1}\left(M_{1}\left(G_{1}\right)-3 \Delta\left(G_{1}\right)\right)\right) \mu^{*}\left(G_{2}\right)
\end{aligned}
$$




$$
\begin{aligned}
& -\mu\left(G_{1}\right) \mu^{*}\left(G_{2}\right)-\mu\left(G_{2}\right) \mu^{*}\left(G_{1}\right)+2 \mu\left(G_{1}\right) \mu\left(G_{2}\right) \\
& -2 n_{2}\left(n_{2}^{2}-4 e_{2}\right) M_{1}\left(G_{1}\right)-2 n_{1}\left(n_{1}^{2}-4 e_{1}\right) M_{1}\left(G_{2}\right) \\
& \left.-2 M_{1}\left(G_{1}\right) M_{1}\left(G_{2}\right)+4\left(e_{1} n_{2}^{2}+e_{2} n_{1}^{2}-2 e_{1} e_{2}\right)^{2}-16 n_{1} n_{2} e_{1} e_{2}\right] .
\end{aligned}
$$

Proof. Let $G=G_{1} \vee G_{2}$. By applying Propositions 1-2, Lemmas 1-2, and definition of the edge-Wiener index $W_{e}(G)$, we get

$$
\begin{aligned}
W_{e}(G) & =\sum_{\{f, g\} \subseteq E(G)} d_{e}(f, g \mid G)=\sum_{\{f, g\} \in A \cup B \cup C} d_{e}(f, g \mid G) \\
& =\sum_{\{f, g\} \in A} d_{e}(f, g \mid G)+\sum_{\{f, g\} \in B} d_{e}(f, g \mid G)+\sum_{\{f, g\} \in C} d_{e}(f, g \mid G) \\
& =|A|+2|B|+3|C|=2(|A|+|B|+|C|)-|A|+|C| .
\end{aligned}
$$

Now, using (11), (12), and (14), we can get (19).

Let $P_{n}$ and $C_{n}$ denote the $n$-vertex path and cycle, respectively. It can be verified by a direct calculation that, for every $n \geqslant 2$,

$$
\begin{aligned}
M_{1}\left(P_{n}\right)=4 n-6 ; \quad \Delta\left(P_{n}\right)=0 ; \\
\nu\left(P_{n}\right)= \begin{cases}4 & \text { if } n=2, \\
16 n-30 & \text { if } n \geqslant 3 ;\end{cases} \\
\nu^{*}\left(P_{n}\right)= \begin{cases}10 & \text { if } n=2, \\
2\left(8 n^{2}-27 n+31\right) & \text { if } n \geqslant 3 ;\end{cases} \\
\mu\left(P_{n}\right)= \begin{cases}0 & \text { if } n=2, \\
2(n-3)(n-4) & \text { if } n \geqslant 3 ;\end{cases} \\
\mu^{*}\left(P_{n}\right)= \begin{cases}0 & \text { if } n=2, \\
2(n-3)\left(n^{2}-6 n+10\right) & \text { if } n \geqslant 3 .\end{cases}
\end{aligned}
$$

Also for every $n \geqslant 3$,

$$
\begin{aligned}
& M_{1}\left(C_{n}\right)=4 n ; \\
& \Delta\left(C_{n}\right)= \begin{cases}1 & \text { if } n=3, \\
0 & \text { if } n \geqslant 4 ;\end{cases} \\
& \nu\left(C_{n}\right)= \begin{cases}27 & \text { if } n=3, \\
16 n & \text { if } n \geqslant 4 ;\end{cases} \\
& \nu^{*}\left(C_{n}\right)= \begin{cases}160 & \text { if } n=4, \\
2 n(8 n-13) & \text { if } n \neq 4 ;\end{cases}
\end{aligned}
$$




$$
\begin{aligned}
& \mu\left(C_{n}\right)= \begin{cases}0 & \text { if } n \leqslant 4, \\
2 n(n-5) & \text { if } n \geqslant 5 ;\end{cases} \\
& \mu^{*}\left(C_{n}\right)= \begin{cases}0 & \text { if } n \leqslant 4, \\
2 n(n-4)^{2} & \text { if } n \geqslant 5 .\end{cases}
\end{aligned}
$$

Now using (19), we easily arrive at:

Corollary 1. For every integers $n \geqslant 2$ and $m \geqslant 3$,

$$
\begin{aligned}
& W_{e}\left(P_{n} \vee C_{m}\right) \\
& =\left\{\begin{array}{lll}
150 & \text { if } n=2, & m=3, \\
432 & \text { if } n=2, & m=4, \\
m\left(m^{3}+3 m^{2}+m-9\right) & \text { if } n=2, & m \geqslant 5, \\
\frac{1}{2}\left(18 n^{4}+24 n^{3}-39 n^{2}-45 n+72\right) & \text { if } n \geqslant 3, & m=3, \\
8\left(2 n^{4}+7 n^{3}-2 n^{2}-30 n+38\right) & \text { if } n \geqslant 3, & m=4, \\
\frac{1}{2} m\left[n^{4}(3 m-5)\right. & \text { if } n \geqslant 3, & m \geqslant 5 . \\
\quad+2 n^{3}\left(3 m^{2}-18 m+44\right) & & \\
\quad+n^{2}\left(3 m^{3}-42 m^{2}+280 m-722\right) & & \\
\quad-n\left(11 m^{3}-152 m^{2}+1038 m-2524\right) &
\end{array}\right.
\end{aligned}
$$

\section{Acknowledgments}

The authors would like to thank the referee for insightful comments and valuable suggestions. The partial support by the Center of Excellence of Algebraic Hyper-structures and its Applications of Tarbiat Modares University (CEAHA) is gratefully acknowledged by the second author.

\section{References}

[1] M. Azari and A. Iranmanesh, Computation of the edge Wiener indices of the sum of graphs, Ars Combin., V. 100, 2011, pp. 113-128.

[2] M. Azari and A. Iranmanesh, Computing Wiener-like topological invariants for some composite graphs and some nanotubes and nanotori, In: I. Gutman, (Ed.), Topics in Chemical Graph Theory, Univ. Kragujevac, Kragujevac, 2014, pp. 69-90.

[3] M. Azari and A. Iranmanesh, The second edge-Wiener index of some composite graphs, Miskolc Math. Notes, V. 15, N. 2, 2014, pp. 305-316.

[4] M. Azari and A. Iranmanesh, Edge-Wiener type invariants of splices and links of graphs, Politehn. Univ. Bucharest Sci. Bull. Ser. A Appl. Math. Phys., V. 77, N. 3, 2015, pp. 143-154. 
[5] M. Azari and A. Iranmanesh, Clusters and various versions of Wiener-type invariants, Kragujevac J. Math., V. 39, N. 2, 2015, pp. 155-171.

[6] M. Azari, A. Iranmanesh and A. Tehranian, Computation of the first edge Wiener index of a composition of graphs, Studia Univ. Babes Bolyai Chem., V. 55, N. 4, 2010, pp. 183-196.

[7] M. Azari, A. Iranmanesh and A. Tehranian, A method for calculating an edge version of the Wiener number of a graph operation, Util. Math., V. 87, 2012, pp. 151-164.

[8] F. Cataldo, O. Ori and A. Graovac, Wiener index of 1-pentagon fullerenic infinite lattice, Int. J. Chem. Model., V. 2, , 2010, pp. 165-180.

[9] P. Dankelman, I. Gutman, S. Mukwembi and H. C. Swart, The edge Wiener index of a graph, Discrete Math., V. 309, 2009, pp. 3452-3457.

[10] M. R. Darafsheh and M. H. Khalifeh, Calculation of the Wiener, Szeged, and PI indices of a certain nanostar dendrimer, Ars Combin., V. 100, 2011, pp. 289-298.

[11] M. V. Diudea, Wiener index of dendrimers, MATCH Commun. Math. Comput. Chem., V. 32, 1995, pp. 71-83.

[12] M. V. Diudea, QSPR/QSAR Studies by Molecular Descriptors, NOVA, New York, 2001.

[13] T. Došlić, Splices, links and their degree-weighted Wiener polynomials, Graph Theory Notes N. Y., V. 48, 2005, pp. 47-55.

[14] T. Došlić, Vertex-weighted Wiener polynomials for composite graphs, Ars Math. Contemp., V. 1, N. 1, 2008, pp. 66-80.

[15] A. Graovac and T. Pisanski, On the Wiener index of a graph, J. Math. Chem., V. 8, 1991, pp. 53-62.

[16] I. Gutman and N. Trinajstić, Graph theory and molecular orbitals, Total $\pi$-electron energy of alternant hydrocarbons, Chem. Phys. Lett., V. 17, 1972, pp. 535-538.

[17] W. Imrich and S. Klavžar, Product Graphs: Structure and Recognition, John Wiley \& Sons, New York, 2000.

[18] A. Iranmanesh and M. Azari, Edge-Wiener descriptors in chemical graph theory: A survey, Curr. Org. Chem., V. 19, N. 3, 2015, pp. 219-239.

[19] A. Iranmanesh, I. Gutman, O. Khormali and A. Mahmiani, The edge versions of the Wiener index, MATCH Commun. Math. Comput. Chem., V. 61, 2009, pp. 663-672.

[20] M. H. Khalifeh, H. Yousefi-Azari and A. R. Ashrafi, The first and second Zagreb indices of some graph operations, Discrete Appl. Math., V. 157, 2009, pp. 804-811.

[21] M. H. Khalifeh, H. Yousefi-Azari, A. R. Ashrafi and S. G. Wagner, Some new results on distance-based graph invariants, European J. Combin., V. 30, 2009, pp. 1149-1163.

[22] M. J. Nadjafi-Arani, H. Khodashenas and A. R. Ashrafi, Relationship between edge Szeged and edge Wiener indices of graphs, Glas. Mat. Ser. III, V. 47, 2012, pp. 21-29.

[23] H. Wiener, Structural determination of paraffin boiling points, J. Am. Chem. Soc., V. 69, N. 1, 1947, pp. 17-20. 
[24] H. Yousefi-Azari, M. H. Khalifeh and A. R. Ashrafi, Calculating the edge Wiener and edge Szeged indices of graphs, J. Comput. Appl. Math., V. 235, N. 16, 2011, pp. $4866-4870$.

\section{CONTACT INFORMATION}

Mahdieh Azari Department of Mathematics, Kazerun Branch, Islamic Azad University, P. O. Box: 73135-168, Kazerun, Iran

E-Mail(s): azari@kau.ac.ir

Ali Iranmanesh Department of Pure Mathematics, Faculty of Mathematical Sciences, Tarbiat Modares

University, P. O. Box: 14115-137, Tehran, Iran E-Mail(s): iranmanesh@modares.ac.ir

Received by the editors: 27.06.2016

and in final form 27.09.2017. 